\title{
REVIEW
}

\section{The clinical conundrum of neonatal seizures}

\section{Levene}

There is increasing evidence that neonatal seizures have an adverse effect on neurodevelopmental progression and may predispose to cognitive, behavioural, or epileptic complications later in life. However, given the uncertainty about the efficacy and toxicity of the commonly used anticonvulsants, when and how aggressively to treat such seizures is a difficult decision.

$\mathrm{N}$ eonatal seizures or electroconvulsive activity are usually the sign of neuronal compromise and the prognosis in large part will depend on any one of the many underlying causes of the seizure activity. Seizures in the neonatal period usually elicit an aggressive therapeutic response with administration of a variety of poorly evaluated drugs. This annotation explores recent evidence that the duration and frequency of the seizures may also have an additional harmful effect on outcome, with the consequence that effective anticonvulsant therapy may improve prognosis.

The incidence of seizures in the neonatal period is considerably higher than at any other time of life. Animal studies have shown that the immature brain is more prone to seizure activity than the mature brain, but paradoxically the immature brain appears to be less vulnerable than the adult brain to neuronal damage as a result of the seizures (see Holmes and Ben-Ari ${ }^{1}$ for review).

The incidence of seizures in the neonatal period is considerably higher than at any other time of life.

The reason for the increased susceptibility of the immature brain to seizure activity is the developmental stage of opposing neuroexcitatory and neuroinhibitory activity, as GABAergic synapses are functionally more active than NMDAAMPA ones and provide a net excitatory drive in the developing brain. In early life, GABA receptors have a mainly excitatory effect. This changes with progressive development when the sensitivity of the brain to seizures reduces.

An important concept of seizure susceptibility in the brain is kindling, and it is considered to be a more important factor in the immature than in the adult brain. Kindling refers to the effect of repeated but brief stimulation on a susceptible area of the brain, which produces an accelerating and prolonged effect resulting in generalised seizures. In a clinical context it has been suggested that kindling due to repeated subclinical stimuli may render a region of neurones epileptic. Repetitive seizures result in both a progressive increase in the duration of seizures and a decrease in the latency between seizures, and it has been shown in animal studies that kindling in the neonatal period results in an increased susceptibility to seizures later in life. ${ }^{2}$

\section{WHAT CAN WE LEARN FROM ANIMAL EXPERIMENTS?}

Until recently it has been difficult to extrapolate from animal studies the significance to the human neonatal brain of experimental seizure activity. Methodological problems included the nature of the experimental convulsions (prolonged seizure activity induced by chemical or electrical stimulation), the developmental age of the experimental animal, and the difficulty in understanding the effect on functional development as opposed to anatomical evidence of neuronal necrosis.

Recent advances in experimental design have thrown important new light on a number of these uncertainties. Frequent short lived convulsions have been produced by placing the neonatal rat pups (postnatal days 0-4) repeatedly in the volatile agent fluorothyl until both clinical and electroencephalographic (EEG) seizure activity was noted when they were withdrawn. This model allows the production of five short lived seizures per day for five days, ${ }^{3}{ }^{4}$ which is much closer to the intermittent seizure disorder seen in premature and term neonates.

This model did not result in neuronal necrosis, ${ }^{4}$ but there was measurable morphological change involving cortical neuronal activation and density when the rat brains were examined in adult life, ${ }^{3}$ as well as significant functional adverse effects on the rats' learning and memory when they reached maturity. In contrast, a single prolonged seizure in the neonatal period does not produce similar effects. ${ }^{5}$ Limited uncontrolled data in humans suggest that neonatal seizures may also have subtle long term adverse effects on various areas of cognitive function in children thought to be without overt neurological damage. ${ }^{6}$ Furthermore, status epilepticus in adolescent animals previously primed by short seizures in the neonatal period produced substantially increased neuronal loss. ${ }^{4}$ Severe convulsions in the adolescent period resulted in more neuronal damage than less prolonged seizures.

In summary, the experimental data support the contention that frequent, relatively short convulsions cause morphological changes in the immature brain which may affect subsequent behaviour. These changes may also increase the susceptibility to further brain damage if subsequent prolonged convulsions develop in later childhood. 


\section{CLINICAL MONITORING TO DETECT NEONATAL SEIZURES}

It is well recognised that there is poor concordance between clinically evident convulsions and electroconvulsive seizures present on EEG monitoring. ${ }^{7}$ Developments in continuous monitoring techniques now provide compressed continuous amplitude integrated EEG monitoring (also referred to as aEEG) in a clinical situation. These techniques have been shown to predict outcome accurately in asphyxiated infants. ${ }^{8-10}$

Continuous aEEG monitoring requires relatively little training in the interpretation of seizure activity lasting 20 seconds or more or in the identification of abnormal interseizure activity, and this equipment is relatively inexpensive. The question is whether these devices should be widely used in clinical practice. In my view, the two main clinical indications are (a) for monitoring the pharmacologically paralysed infant to detect seizure activity that would be missed clinically and (b) for the prediction of outcome in neurologically compromised mature infants. The role of routine aEEG in premature non-paralysed infants is far less certain and requires further investigation. It may prove useful in infants who are showing equivocally abnormal movements but there is uncertainty as to whether they represent seizure activity. The trace may reduce or increase the threshold for anticonvulsant treatment.

\section{MANAGEMENT}

The recent experimental work showing that short lived seizures in immature animals may have a long term effect on cerebral function swings the threshold further towards appropriate pharmacological treatment of these events. The risk of seizure recurrence in children who have sustained neonatal convulsions is reported to be $8-20 \%,{ }^{11}{ }^{12}$ and those with more frequent seizures are most likely to have postneonatal convulsions. ${ }^{11}$ As prognosis is at least partly dependent on the cause of the seizures, brain imaging is particularly useful in determining an underlying cause, although considerably more than half of babies with seizures will show no structural abnormality when standard imaging techniques are used..$^{13}$

Unfortunately, treatment of neonatal seizures has changed little over the last 50 years and has been the subject of remarkably few good research studies in a clinical setting. Phenobarbitone remains the mainstay of management of neonatal convulsions, but the efficacy of this drug remains uncertain. There have been very few clinical trials in the management of the neonate, and these suggest at best a partial effect in the suppression of neonatal convulsions. ${ }^{14-16}$ Painter and colleagues ${ }^{14}$ compared the efficacies of phenobarbitone and phenytoin in the management of EEG diagnosed neonatal seizures. This was a single blinded crossover study, and the drug dose was maintained in the mid-therapeutic range in both groups. In less than $50 \%$ of either group were the convulsions completely controlled, and this proportion only increased slightly to $59 \%$ when the crossover anticonvulsant drug was added. Those infants with the least severe seizures were the ones in whom the anticonvulsant treatment was most successful. Others have shown that high dose phenobarbitone $(40 \mathrm{mg} / \mathrm{kg})$ given to asphyxiated term newborn infants at risk of developing convulsions significantly reduced severe neurodevelopmental disability or death in the treated group compared with those who were given phenobarbitone once seizures were evident. ${ }^{17}$ A Cochrane review of anticonvulsants in the neonatal period in the prevention of mortality and morbidity did not confirm this benefit. ${ }^{15}$
Phenobarbitone has been reported to have adverse effects on the developing brain, including inhibition of brain growth, ${ }^{18}$ neuronal toxicity, ${ }^{19}$ and adverse cognitive and behavioural effects when administered to young animals. ${ }^{20}$ Phenytoin shows unpredictable plasma levels and cardiotoxicity. Other anticonvulsants used to treat refractory neonatal seizures include the benzodiazepines (clonazepam, ${ }^{21}$ midazolam, ${ }^{22}{ }^{23}$ lorazepam $^{24}$ ) as well as lignocaine, ${ }^{25}$ thiopentone, ${ }^{26}$ sodium valproate, ${ }^{27}$ and lamotrigine. ${ }^{28}$ These reports are largely anecdotal and it is not possible to evaluate their relative efficacy.

\section{A PRAGMATIC APPROACH}

There is increasing evidence that neonatal seizures have a subtle but measurable adverse effect on neurodevelopmental progression and may predispose to cognitive, behavioural, or epileptic complications later in life. Modern and relatively simple devices such as the aEEG will allow earlier and more accurate recognition of electroconvulsive seizures and help to determine whether ambiguous clinical movements represent convulsions or not.

Unfortunately, we also know of no truly effective neonatal anticonvulsant while being concerned that phenobarbitone, the most widely used first line anticonvulsant, may actually compromise outcome because of its toxicity within the nervous system. How to respond to this dilemma of when to treat remains a major therapeutic conundrum.

It is my experience that neonatal convulsions tend to be overtreated in an attempt to abolish all evidence of seizure activity. As shown above, those babies with the most frequent seizures will be the ones most resistant to anticonvulsants. To resolve the problem of which anticonvulsants should be used to treat neonatal convulsions and at what dose, we must insist on more randomised controlled studies with reports on subsequent long term follow up. Without this evidence based data, the question cannot be definitively answered.

It is my practice to try to keep the number of different anticonvulsant drugs used in the management of neonatal seizures to a minimum. Prolonged neonatal seizures (longer than one minute) or frequent seizures (more than two in one hour) should be aggressively treated with phenobarbitone up to a maximum loading dose of $30 \mathrm{mg} / \mathrm{kg}$ in divided doses followed by a one off loading dose of phenytoin $(20 \mathrm{mg} / \mathrm{kg})$. Continued seizures may be best controlled by a continuous infusion of midazolam, which is becoming more widely used in the sedation of babies and children given intensive care and appears to have good safety margins. Seizures should not result in junior medical staff adding further and further anticonvulsants to the baby's medication, but each neonatal unit should develop a drug protocol based on a controlled and graded response to continuing convulsions.

The risk of seizure recurrence in children with neonatal convulsions is relatively small, ${ }^{11}{ }^{12}$ and, taking into account the potential toxicity of some anticonvulsant drugs, it seems good practice to stop anticonvulsant treatment as early as possible. My own practice is to withdraw anticonvulsants before discharge home if the baby is clinically neurologically normal on examination. In those that are discharged home on anticonvulsant treatment, withdrawal is undertaken at the earliest opportunity when clinical neurological recovery occurs. In persistently neurologically abnormal infants, anticonvulsants may be continued for up to six months but an attempt should be made to withdraw phenobarbitone by that time, and, if seizures recur, a more appropriate anticonvulsant should be introduced

\section{Author's affiliation}

M Levene, Academic Unit of Paediatrics, Obstetrics and Gynaecology, University of Leeds, D Floor, Clarendon Wing, Leeds LS2 9NS, UK 


\section{REFERENCES}

1 Holmes GL, Ben-Ari Y. Seizures of the developing brain: perhaps not so benign after all. Neuron 1998;21:1231-4.

2 Moshe SL, Albala BJ. Kindling in developing rats: persistence of seizures into adulthood. Dev Brain Res 1982;4:67-71.

3 Holmes GL, Gairsa JL, Chevassus-Au-Louis N, et al. Consequences of neonatal seizures in the rat: morphological and behavioural effects. Ann Neurol 1998:44:845-57.

4 Schmid R, Tandon P, Stafstrom CE, et al. Effects of neonatal seizures on subsequent seizure-induced brain injury. Neurology 1999;53:1754-61.

5 Stafstrom CE, Chronopoulos A, Thurber S, et al. Age-dependent cognitive and behavioural deficits following kainic acid-inducing seizures. Epilepsia 1993;34:420-32

6 Temple CM, Dennis J, Carney R, et al. Neonatal seizures: long-term outcome and cognitive development among 'normal' survivors. Dev Med Child Neurol 1995;37:109-18.

7 Mizrahi EM, Kellaway P. Characterization and classification of neonatal seizures. Neurology 1987;37:1837-44.

8 Hellstrom-Westas L, Rosen I, Svenningsen NW. Predictive value of early continuous amplitude integrated EEG recordings on outcome after severe birth asphyxia in full term infants. Arch Dis Child 1995:72:F34-8.

9 Eken P, Toet MC, Groenendaal F, et al. Predictive value of early neuroimaging, pulsed Doppler and neurophysiology in full term infants with hypoxic-ischaemic encephalopathy. Arch Dis Child Fetal Neonatal Ed 1995;73:F75-80

10 al Naqeeb N, Edwards AD, Cowan FM, et al. Assessment of neonatal encephalopathy by amplitude-integrated electroencephalography. Pediatrics 1999;103:1263-71.

11 Hellström-Westas L, Blennow $G$, Lindroth $M$, et al. Low risk of seizure recurrence after early withdrawal of antiepileptic treatment in the neonatal period. Arch Dis Child Fetal Neonatal Ed 1995;72:F97-101.

12 Holden KR, Mellits ED, Freeman JM. Neonatal seizures. I. Correlation of prenatal and perinatal events with outcome. Pediatrics 1982;70:165-76.

13 Levene MI, Trounce JQ. Cause of neonatal convulsions: towards more precise diagnosis. Arch Dis Child 1986;61:78-9.
14 Painter MJ, Scher MS, Stein AD, et al. Phenobarbital compared with phenytoin for the treatment of neonatal seizures. N Engl J Med 1999;341:485-9.

15 Evans DJ, Levene MI. Anticonvulsants for preventing mortality and morbidity in full term newborns with perinatal asphyxia. The Cochrane Database of Systematic Reviews 2001; 1 :review.

16 Boylan G, Rennie JM, Pressler R, et al. Evaluation of phenobarbitone treatment for neonatal seizures. Arch Dis Child 2001;84(suppl I):A3

17 Hall RT, Hall FK, Daily DK. High-dose phenobarbital therapy in term newborn infants with severe perinatal asphyxia: a randomised, prospective study with three-year follow-up. J Pediatr 1998;132:345-8.

18 Diaz J, Schain J, Bailey BG. Phenobarbital-induced brain growth retardation in artificially reared rat pups. Biol Neonate 1977;32:77-82.

19 Mattson RH. Phenobarbital toxicity. In: Levy RH, Dreifuss FE, Mattson RH, eds. Antiepileptic drugs. 3rd ed. New York: Raven, 1985:341-55.

20 Mikati MA, Holmes GL, Chonopoulos A, et al. Phenobarbital modifies seizure-related brain injury in the developing brain. Ann Neurol 1994; 36:425-33.

21 Andre M, Boutry M, Dubroc C. Clonazepam pharmacokinetics and therapeutic efficacy in neonatal seizures. Eur J Clin Pharmacol 1986:30:585-9.

22 Holmes GL, Riviello JJ. Midazolam and pentobarbital for refractory status epilepticus. Pediatr Neurol 1999;20:259-64.

23 Gherpelli JLD, Lucas FJC, Roitman I, et al. Midazolam for treatment of refractory neonatal seizures. Arch Neuropsychiatry 1994;52:260-2.

24 Maytal J, Novak GP, King KC. Lorazepam in the treatment of refractory neonatal seizures. J Child Neurol 1991;6:319-23.

25 Hellstrom-Westas L, Westgren U, Rosen I, et al. Lidocaine for treatmen of severe seizures in newborn infants. I. Clinical effects and cerebral electrical activity monitoring. Acta Paedaitr Scand 1988;77:79-84.

26 Bonati M, Marraro G, Celardo A. Thiopental efficacy in Phenobarbital-resistant neonatal seizures. Dev Pharmacol Ther 1990;15:16-20

27 Gal P, Oles KS, Gilman JT, et al. Valproic acid efficacy, toxicity, and pharmacokinetics in neonates with intractable seizures. Neurology 1988;38:467-71.

28 Barr PA, Buettiker VE, Antony JH. Efficacy of lamotrigine in refractory neonatal seizures. Pediatr Neurol 1999;20:161-3.

\section{This month in the Archives of Disease in Childhood}

The following papers appearing in the March 2002 issue of $A D C$ may be of interest to readers of Fetal and Neonatal.

Maternal compliance with nutritional recommendations in an allergy preventive programme. A Schoetzau, U Gehring, K Franke, et al.

An evaluation of the first parent health visitor scheme. A Emond, J Pollock, T Deave, et al.

Vitamin D deficiency in UK Asian families: activating a new concern. N J Shaw, B R Pal.

Controversy: Plagiocephaly and head binding. S J Bridges, T L Chambers, I K Pople, with commentary by S A Wall. 